\title{
Compact, passively Q-switched, all-solid-state master oscillator-power amplifier-optical parametric oscillator (MOPA-OPO) system pumped by a fiber-coupled diode laser generating high-brightness, tunable, ultraviolet radiation
}

\author{
Peter Peuser, ${ }^{1, \star}$ Willi Platz, ${ }^{1}$ Andreas Fix, ${ }^{2}$ Gerhard Ehret, ${ }^{2}$ Alexander Meister, ${ }^{3}$ \\ Matthias Haag, ${ }^{4}$ and Paul Zolichowski ${ }^{4}$ \\ ${ }^{1}$ EADS Innovation Works, IW-SI, P.O. Box 800465, 81663 München, Germany \\ ${ }^{2}$ Deutsches Zentrum für Luft- und Raumfahrt (DLR), Institut für Physik der Atmosphäre, \\ 82234 Oberpfaffenhofen, Germany \\ ${ }^{3}$ Kayser-Threde $\mathrm{GmbH}$, Wolfratshauser Strasse 48, 81379 München, Germany \\ ${ }^{4}$ Dilas GmbH, Galileo-Galilei-Strasse 10, 55129 Mainz-Hechtsheim, Germany \\ ${ }^{*}$ Corresponding author: peter.peuser@eads.net \\ Received 17 February 2009; revised 26 May 2009; accepted 12 June 2009; \\ posted 17 June 2009 (Doc. ID 107479); published 29 June 2009
}

\begin{abstract}
We report on a compact, tunable ultraviolet laser system that consists of an optical parametric oscillator (OPO) and a longitudinally diode-pumped Nd:YAG master oscillator-power amplifier (MOPA). The pump energy for the whole laser system is supplied via a single delivery fiber. Nanosecond pulses are produced by an oscillator that is passively $Q$-switched by a $\mathrm{Cr}^{4+}$ :YAG crystal. The OPO is pumped by the second harmonic of the Nd:YAG MOPA. Continuously tunable radiation is generated by an intracavity sumfrequency mixing process within the OPO in the range of $245-260 \mathrm{~nm}$ with high beam quality. Maximum pulse energies of $1.2 \mathrm{~mJ}$ were achieved, which correspond to an optical efficiency of $3.75 \%$, relating to the pulse energy of the MOPA at $1064 \mathrm{~nm}$. (C) 2009 Optical Society of America

OCIS codes: $\quad 140.3480,140.3280,140.3580,190.4970,140.3600,140.3610$.
\end{abstract}

\section{Introduction}

Compact, tunable, nanosecond-pulse, all-solid-state laser systems operating at ultraviolet wavelengths are of fundamental interest for applications in lidar techniques [1] and resonant ionization spectrometry [2], as well as for atmospheric ultraviolet spectroscopy, chemical or biological hazard detection, laser-induced fluorescence spectroscopy, combustion diagnostics, optogalvanic spectroscopy, and photobiology [3].

0003-6935/09/193839-07\$15.00/0

(C) 2009 Optical Society of America
Recently, laser-based ion mobility spectrometry [4] achieved special appreciation since, by this method, practical detection systems become available with high usefulness for the sensitive and selective detection of aromatic chemical compounds. To meet the requirements of mobile and versatile measuring systems, small and robust, tunable UV lasers are needed that are able to generate nanosecond pulses with energies in the millijoule range and a high efficiency that would allow battery driven operation. Moreover, high beam quality is especially important with respect to achieving efficient interaction with the molecules to be sensed. The goal of this work is to investigate and develop a laser that is 
characterized by such features. It comprises a compact optical parametric oscillator (OPO) with intracavity sum-frequency mixing and a Nd:YAG master-oscillator-power amplifier (MOPA), which is longitudinally diode pumped using energy transportation via an optical fiber. The OPO is pumped by the second harmonic of the Nd:YAG MOPA. Pulses in the nanosecond range are generated by an oscillator that is passively $Q$-switched by a $\mathrm{Cr}^{4+}$ crystal.

The usefulness of sum-frequency generation to produce tunable UV radiation from nanosecond solid-state laser sources was demonstrated by several authors for alexandrite lasers [5-7], Ti:sapphire lasers [8-14], Cr-doped colquiriite lasers [15], or OPOs [16-20] in the past. However, none of the reported configurations would be able to fulfill the requirements of a laser for a compact, versatile ion mobility spectrometer. The same holds for directly pumped tunable UV lasers, such as Ce-doped lasers, which are generally pumped using the fourth harmonic of Nd:YAG lasers and produce tunable output in the 280 to $330 \mathrm{~nm}$ spectral region [3].

The laser system described in the following represents a combination of previous works of the authors on OPO technology with intracavity sum-frequency generation [21] and on fiber-coupled, diode-pumped MOPA configurations [22], with the special goals of miniaturizing overall size, weight, energy consumption, and cooling requirements. In these works it was demonstrated that intracavity sum-frequency mixing provides high optical conversion efficiency for the generation of UV radiation together with high beam quality, whereas longitudinal diode pumping of a MOPA in combination with passive $Q$-switching allows constructing a compact, efficient, robust laser source for the OPO. The OPO configurations investigated in [21] were pumped by the second harmonic of a Nd:YAG laser at $532 \mathrm{~nm}$ and were operated at pulse energies in the range between a threshold of about 30 and $200 \mathrm{~mJ}$. This required high pulse energies of approximately $400 \mathrm{~mJ}$ for the flashlamp-pumped $\mathrm{Nd}$ : YAG laser. Therefore, it was a special goal of our work to realize an OPO with a significantly reduced threshold in order to afford the use of a small, robust, diode-pumped Nd:YAG laser providing maximum pulse energies of about $30 \mathrm{~mJ}$ only. This would allow us to build an efficient, compact, tunable UV-laser system and demonstrate its utility for mobile or airborne applications. Thus, another OPO design was required.

The first application of the laser system was intended for an ion mobility spectrometer in order to achieve efficient ionization of aromatic molecules in the wavelength region around $250 \mathrm{~nm}$. The complete laser setup was planned not to exceed a footprint of $45 \mathrm{~cm} \times 45 \mathrm{~cm}$ and an overall height of $10 \mathrm{~cm}$. This condition, first of all, made it necessary to develop a Nd:YAG MOPA with much smaller dimensions than those described in [22]. This meant a smaller amplifier stage in particular, and that required some effort to circumvent optical feedback into the laser oscillator as well as parasitic lasing of the amplifier caused by extremely high inversion densities within such a compact optomechanical configuration. Multipass MOPA configurations as described in [23] would not be appropriate for our system. In that case, right-angle prisms or corner cubes are used to form the amplifier beam path in combination with a lens, which focuses the radiation of the multiple amplifier branches onto a reflecting pump mirror. In contrast to that paper, the pump power applied for our setup, as well as the laser beam intensity, is higher by orders of magnitude, which would lead to exceeding the damage threshold of the reflecting mirror and cause strong optical feedback and parasitic lasing. Furthermore, a compact and efficient nonlinear-optical device was needed to generate the radiation for pumping the OPO as well as for intracavity mixing. In addition, an optical delay line had to be integrated to match the OPO signal pulse and the pulse of the mixing beam. Finally, the setup of the tunable OPO had to be assembled on the breadboard.

\section{Experimental Layout}

The scheme of the laser system is shown in Fig. 1 . The energy for the whole laser is supplied by a compact, fiber-coupled, high-power diode module (DM), custom-made by Dilas $\mathrm{GmbH}$, which contains two polarization coupled, stacked arrays of quasi-cw GaAlAs diode bars and a miniaturized fiber coupling optics. The quartz fiber (F) has a core diameter of $800 \mu \mathrm{m}$ and a numerical aperture of 0.22 . About $70 \%$ of the diode power is available at the fiber end, providing a maximum quasi-cw pump power of $1 \mathrm{~kW}$ at a wavelength of $807 \mathrm{~nm}$ for the laser system. During the investigations in the laboratory, a water chiller was used for cooling the diode lasers and controlling the temperature, although this is not mandatory because of the low cooling requirements. As the diode module can be operated up to a duty cycle of $3 \%$, maximum pulse repetition rates in the range of about $100 \mathrm{~Hz}$ are possible for operating a Nd:YAG laser. In our investigations all measurements were performed at a pulse repetition rate of $20 \mathrm{~Hz}$, while the pump pulse duration in general was $300 \mu \mathrm{s}$. The diode radiation is collimated by a lens system (LS1). About $20 \%$ of this beam is reflected by a partially transmitting mirror (M1) for pumping the laser oscillator, while the remaining part is used to feed an amplifier stage via a highly reflecting $45^{\circ}$ mirror (M2). Following the branch of the laser oscillator, another lens system (LS2) focuses the pump beam into a Nd:YAG crystal with a diameter of $3 \mathrm{~mm}$ and a length of $10 \mathrm{~mm}$, which has plane-parallel end faces that are appropriately coated for end pumping, with a transmittance of 98\% at the pump wavelength and a reflectivity of $99.9 \%$ at the laser wavelength of $1064 \mathrm{~nm}$ (M3). The second face has an antireflection (AR) coating at the laser wavelength. A diaphragm (D) with variable aperture serves to control the pump power. 


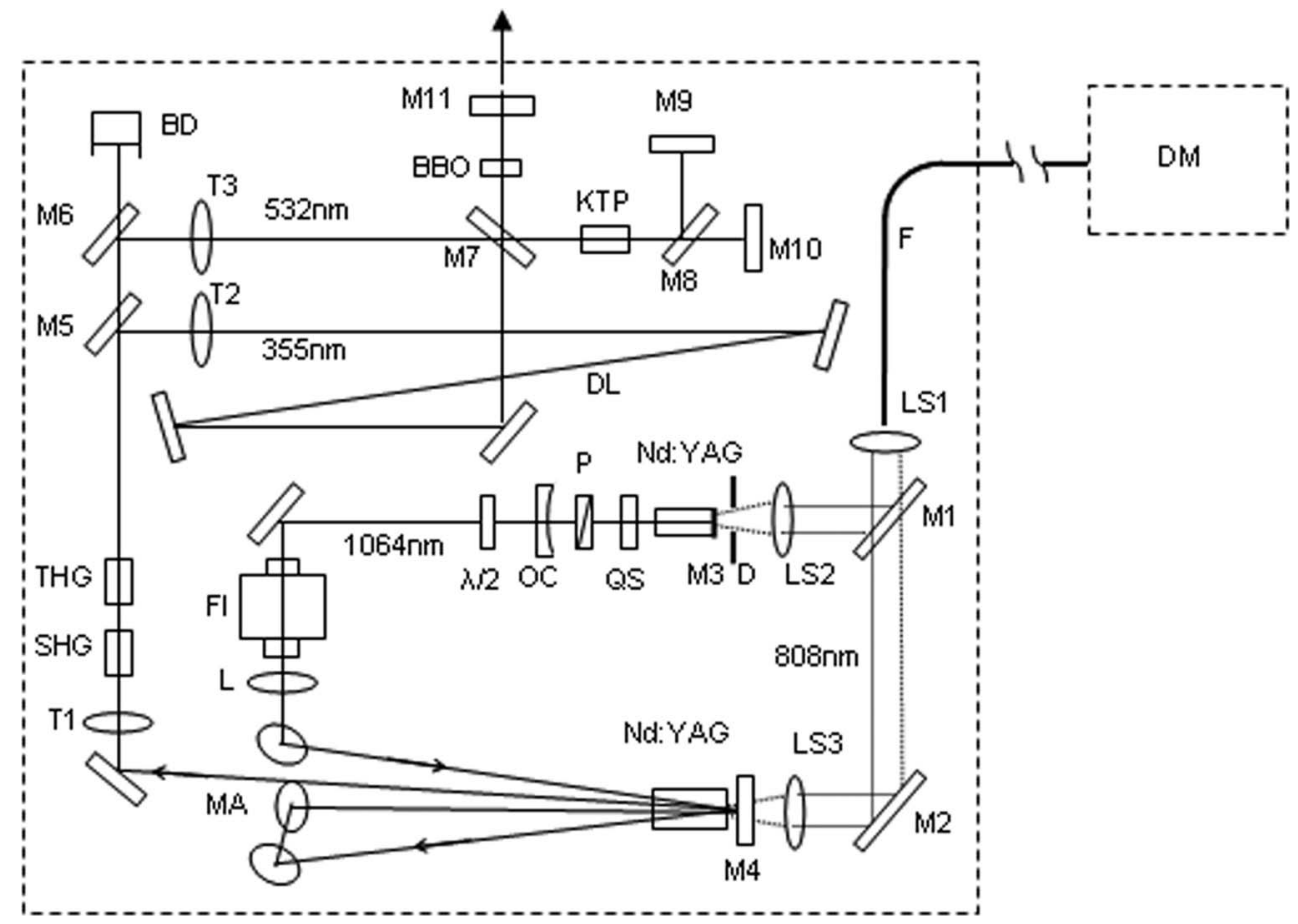

Fig. 1. Setup of the laser system on a $45 \mathrm{~cm} \times 45 \mathrm{~cm}$ breadboard. DM, diode laser module; F, optical fiber; M1, partially reflecting $45^{\circ}$ mirror; M2, highly reflecting $45^{\circ}$ mirror; LS1-LS3, lens systems; D, diaphragm; M3, pump mirror of laser oscillator; QS, passive $Q$-switch crystal; P, polarizer; OC, outcoupling laser mirror; $\lambda / 2$, half-wave plate; FI, Faraday isolator; L, lens; M4, pump mirror of amplifier; MA, mirror configuration of amplifier; T1-T3, telescopes; SHG, crystal for second harmonic generation; THG, crystal for third harmonic generation; M5, M6, dichroic mirrors; BD, beam dump; DL, delay line; M7-M11, mirrors of OPO setup; KTP, OPO crystal; BBO, mixing crystal.

This component is necessary to adjust the onset of the oscillator pulse in relation to the pump pulse independently from the pump current, in order to achieve maximum amplification [22]. The resonator has a length of $90 \mathrm{~mm}$ and is formed by an outcoupling mirror (OC) with a radius of curvature of $2 \mathrm{~m}$ and a reflectivity of $60 \%$ at the laser wavelength. A polarizer $(\mathrm{P})$ is inserted to achieve a stable, linear polarization. Passive $Q$-switching is accomplished by an AR-coated $\mathrm{Cr}^{4+}: \mathrm{YAG}$ crystal (QS) which has a small signal transmission of $30 \%$.

Before entering the amplifier stage the laser beam passes a half-wave plate $(\lambda / 2)$ and a Faraday isolator (FI). A focusing optical lens (L) with $f=1 \mathrm{~m}$ serves to provide an optimum overlap of the oscillator beam with the excited volume in the amplifier crystal. The pump radiation for the amplifier is focused by a lens system (LS3) through a pump mirror (M4) into the $\mathrm{Nd}$ :YAG crystal, resulting in a gain region diameter of about $1.2 \mathrm{~mm}$. The amplifier crystal is AR coated for end pumping. It has a diameter of $6 \mathrm{~mm}$ and a length of $20 \mathrm{~mm}$. The outcoupling end face of the amplifier crystal is cut at an angle of $1^{\circ}$ with respect to the pumped end face to increase the threshold of parasitic lasing. The amplifier stage is characterized by a four-pass geometry, which is generated by the pump mirror in combination with a multimirror configuration (MA). The threedimensional beam path consists of two V-shaped branches that are orthogonal to each other, with their tips on the pump mirror. The lengths of the corresponding four legs were restricted to about $20 \mathrm{~cm}$ in order to save sufficient free space for the wavelength converting part of the laser system. In comparison with the amplifier setup reported in [22], this meant a reduction of the distance between the multimirror system and the amplifier crystal, which eventually would cause severe problems with parasitic lasing of the amplifier. By increasing the apex angle of the V-shaped beam paths to a value of $8^{\circ}$, it was observed that the threshold of parasitic lasing for the amplifier raised to a value higher than $100 \mathrm{~kW} / \mathrm{cm}^{2}$, which is the maximum applied pump intensity for the amplifier. It is assumed that the amount of the laser radiation that is backscattered from the external mirrors (MA) and enters the amplifier crystal is reduced when these mirrors are mounted farther from the central axis defined by the amplifier crystal.

A telescope (T1) with a magnification of $2: 1$ is used to provide optimum beam conditions for the following nonlinear-optical chain. It is placed in front of a closely arranged pair of a frequency-doubling KTP crystals, cut for type II phase matching $\left(\theta=90^{\circ}\right.$, $\varphi=23^{\circ}$ ), and a BBO crystal, cut for type I frequency tripling $\left(\theta=32^{\circ}\right)$, both with an aperture of $7 \mathrm{~mm} \times$ $8 \mathrm{~mm}$ and a length of $12 \mathrm{~mm}$. The $532 \mathrm{~nm}$ beam for the OPO and the $355 \mathrm{~nm}$ beam for the frequencymixing process are separated by means of two dichroic beam splitters (M5, M6), while the unconverted fundamental radiation is transmitted into a 
beam dump (BD). The UV beam passes a telescope (T2), which reduces its diameter of about $1.5 \mathrm{~mm}$ by $1: 1.2$ to match the cross section to that of the intracavity signal beam. A delay line (DL) for the $355 \mathrm{~nm}$ pulse having a length of $50 \mathrm{~cm}$ had to be assembled to compensate for the delay of the OPOsignal pulse, due to its buildup time of about $1.5 \mathrm{~ns}$. Another telescope (T3) is used to reduce the diameter of the green pump beam by a factor of $1: 1.5$, which gave the best results for the operation of the signal-resonant OPO. The width of the tuning range is primarily defined by the optical mirror coatings, which have to simultaneously fulfill the multiple conditions of high reflectivity for the signal wave and high transmittances for the pump radiation, the mixing radiation, and for the resulting UV beam. The resonator mirrors of the OPO were designed to provide a maximum width of the wavelength interval for the signal wave, which was calculated to vary within an interval of 792-965 $\mathrm{nm}$ for achieving UV radiation in the region between about 245 and $260 \mathrm{~nm}$ by sum-frequency mixing with $355 \mathrm{~nm}$. The parametric process is of type II, for which a corresponding KTP crystal was chosen that had an aperture of $8 \mathrm{~mm} \times$ $7 \mathrm{~mm}$ and a length of $15 \mathrm{~mm}$. To fulfill the phasematching conditions, the crystal is cut at an angle of $68^{\circ}$ in the $x z$ plane of the crystal. It is AR coated for the signal and the pump waves. For sumfrequency mixing, an AR-coated BBO crystal is used, which is cut at an angle of $47^{\circ}$ for type I phase matching. It has an aperture of $8 \mathrm{~mm} \times 7 \mathrm{~mm}$ and a length of $3 \mathrm{~mm}$.

In the L-shaped OPO configurations described in [21] the rotation of the plane-parallel OPO crystal during wavelength tuning causes a displacement of the signal beam in the mixing crystal of about $3.5 \mathrm{~mm}$ for the full spectral range. If the beam diameter is large, i. e., several millimeters, as in that case, it does not noticeably influence the mixing process. However, for a much smaller signal beam, as is the case here-since the low pump energy requires a small beam diameter of $1.5 \mathrm{~mm}$ to achieve the OPO threshold intensity-the situation is somewhat different. We therefore devised a U-shaped cavity by which this displacement is avoided because the signal beam passes through the KTP crystal twice before it enters the BBO crystal, with the consequence that the optimum overlap between signal and $355 \mathrm{~nm}$ radiation is maintained during wavelength tuning.

The compact, U-shaped, folded OPO resonator has a geometric length of $100 \mathrm{~mm}$. It is formed between two $45^{\circ}$ reflecting mirrors (M7, M8) and two end mirrors (M9, M11), which have a high reflectivity $(>99 \%)$ for the wavelength range of the OPO signal. The reflectivity at the idler wavelength was calculated to be less than $20 \%$. One of the end mirrors (M11), which has a transmittance of $85 \%$ for the UV radiation, is used to extract the UV beam. The green pump beam enters the resonator through one of the $45^{\circ}$ reflecting mirrors, which has a high transmittance $(>95 \%)$ at $532 \mathrm{~nm}$. This pump mirror (M7) is simultaneously used for coupling the beam for the sum-frequency generation into the resonator and, therefore, also has a high transmittance ( $>95 \%)$ at $355 \mathrm{~nm}$. An external mirror (M10) is used for reflecting the pump radiation through the second $45^{\circ}$ reflecting mirror (M8) back into the resonator, in order to decrease the OPO threshold and to increase the efficiency by double-pass pumping. Wavelength tuning was performed by using two computercontrolled, motorized actuators for rotating both the KTP and the BBO crystal. The rotating axes are perpendicular to each other due to the polarization and the orientation of the two crystals.

\section{Measurements and Results}

The laser oscillator generated pulses with a length of about $4.5 \mathrm{~ns}$ and an energy of $1.5 \mathrm{~mJ}$. After amplification a maximum energy of $38 \mathrm{~mJ}$ was achieved at a diode current of $95 \mathrm{~A}$, which corresponds to a diode pump energy of $207 \mathrm{~mJ}$ (Fig. 2). The longitudinal pump geometry provided virtually constant output energy over a broad temperature range of the pump diodes. This is demonstrated in Fig. 3, where pulse energies at a level of $32 \mathrm{~mJ}$ were achieved at a constant diode current of $80 \mathrm{~A}$. The pump energy delivered by the fiber at this diode current is $166 \mathrm{~mJ}$, which leads to an optical efficiency of $19.2 \%$ for the MOPA, or of $13.4 \%$ when the fibercoupling efficiency for the diodes is taken into account. By operating the MOPA at this output energy, where the $M^{2}$ factor of the laser beam was about 1.5 , the compact frequency converter stage was adjusted to achieve optimum performance of the OPO by tuning the simultaneously produced pulse energies for pumping the OPO and for intracavity sum-frequency generation. Optimum operation was reached for pulse energies of $8.8 \mathrm{~mJ}$ at $532 \mathrm{~nm}$ and of $4.8 \mathrm{~mJ}$ at $355 \mathrm{~nm}$. The corresponding pulse widths were measured to be slightly less than $4 \mathrm{~ns}$. A similar value was also determined, within an uncertainty of about $0.3 \mathrm{~ns}$, for the generated tunable UV radiation, which

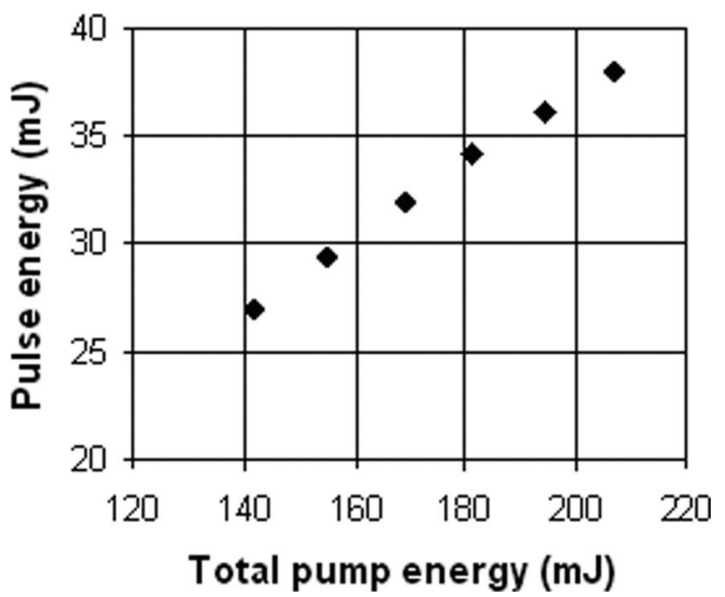

Fig. 2. Pulse energy of the Nd:YAG MOPA as a function of the total pump energy. 


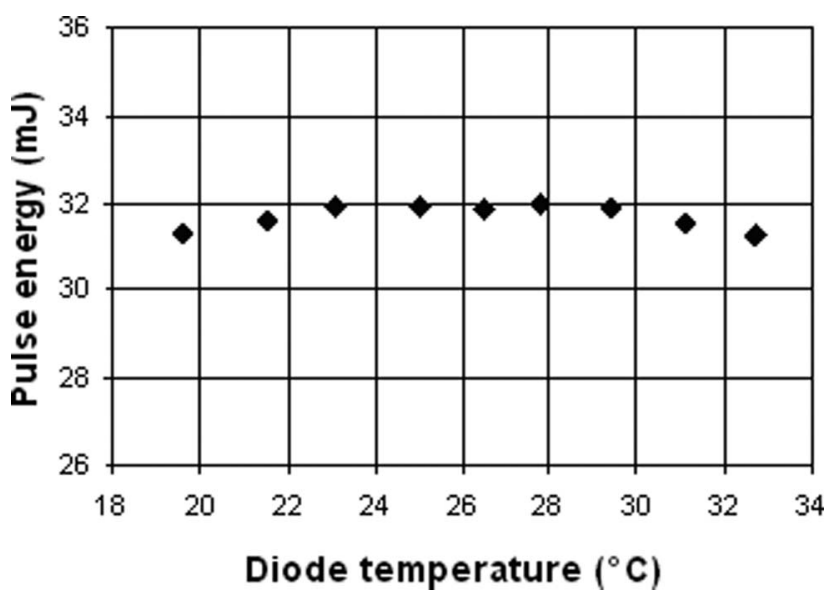

Fig. 3. Pulse energy of the Nd:YAG MOPA as a function of the diode temperature at a constant diode current of $80 \mathrm{~A}$.

is orthogonally polarized with respect to the $355 \mathrm{~nm}$ radiation.

The performance of the OPO was first optimized by carefully adjusting the resonator and observing the small amount of the residual signal radiation that was transmitted by the end mirrors, while the wavelength was determined by means of a miniaturized spectrometer (Ocean Optics) with $1 \mathrm{~nm}$ resolution. Then the third harmonic beam was coupled into the resonator and the $\mathrm{BBO}$ crystal. The UV radiation generated by the mixing process was separated from the rest of the $355 \mathrm{~nm}$ radiation by means of a double stage filter. By preparing an optimum overlap with the signal radiation, the pulse energy was maximized, while a thermopile power meter was used for measuring the pulse energy. The UV wavelength was determined by a double monochromator spectrometer (Jobin Ivon), which has a resolution of $0.1 \mathrm{~nm}$. The OPO threshold was reached at a pump energy of $3.8 \mathrm{~mJ}$, which corresponds to a pump intensity in the range of $50 \mathrm{MW} / \mathrm{cm}^{2}$. A maximum pulse energy of $1.2 \mathrm{~mJ}$ was measured for a wavelength of $256 \mathrm{~nm}$ (Fig. 4). This value was reached after carefully re-

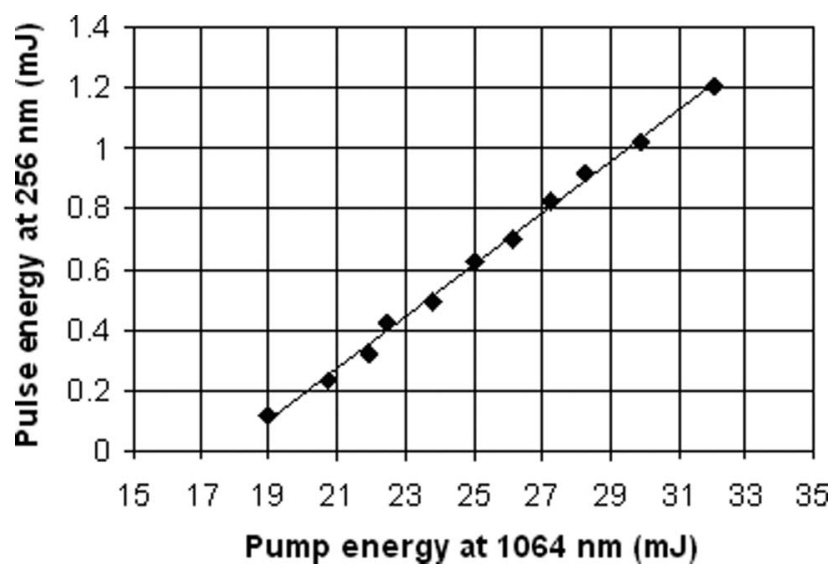

Fig. 4. Pulse energy of the UV-OPO, measured at a wavelength of $256 \mathrm{~nm}$, as a function of the pump energy of the Nd:YAG MOPA. The straight line is from a linear data fit. adjusting the OPO. It corresponds to an optical efficiency of $3.75 \%$ relating to the pulse energy of the $\mathrm{Nd}$ : YAG pump laser system at $1064 \mathrm{~nm}$. The straight line in Fig. 4 represents a linear data fit, which yields a differential efficiency of $8.5 \%$. By taking into account the optical efficiency of $13.4 \%$ of the Nd:YAG MOPA and an electrical-to-optical efficiency of $50 \%$ of the diode lasers, a total efficiency of $0.25 \%$ is calculated for the UV-laser system. Correspondingly, the electrical power consumption at the chosen pulse repetition rate of $20 \mathrm{~Hz}$ is about $9.6 \mathrm{~W}$. By including an efficiency of $85 \%$ of the power control unit and by using passive cooling, the total power consumption is less than $11.5 \mathrm{~W}$.

When the OPO was continuously tuned, without applying any readjustments, the tuning range reached from 245 to $259.5 \mathrm{~nm}$ (Fig. 5), which is primarily limited by the reflection coatings of the OPO mirrors. The linewidth of the generated UV radiation is mainly determined by the width of the OPO-signal radiation, which is of the order of $3 \mathrm{~cm}^{-1}$ (corresponding to less than $0.3 \mathrm{~nm}$ ). In addition, it is rather constant over the respective wavelength range since the OPO process is of type II and, thus, the gain bandwidth is only weakly dependent on wavelength. Because of energy conservation, the UV linewidths (in units of wavenumbers) are comparable to the signal and, therefore, are of the order of $0.02 \mathrm{~nm}$. It has also been shown in the preceding work [1] that the linewidths in the UV are fairly constant over a wide range of energies. The pulse-to-pulse stability was determined to be about $\pm 5 \%$.

Within the scope of this work a detailed investigation on the determination of $M^{2}$ was not performed. However, the $1 / e^{2}$ diameter of the UV beam was measured at a distance of about $1.5 \mathrm{~m}$ behind the OPO, giving a qualitative description of the twodimensional beam profile, instead. This is depicted in Fig. 6, where the beam profile of the UV radiation is presented in comparison with the beam profile of the Nd:YAG MOPA. In addition, intensity curves are shown for horizontal and vertical, central cross sections. The central intensity curves of the UV beam

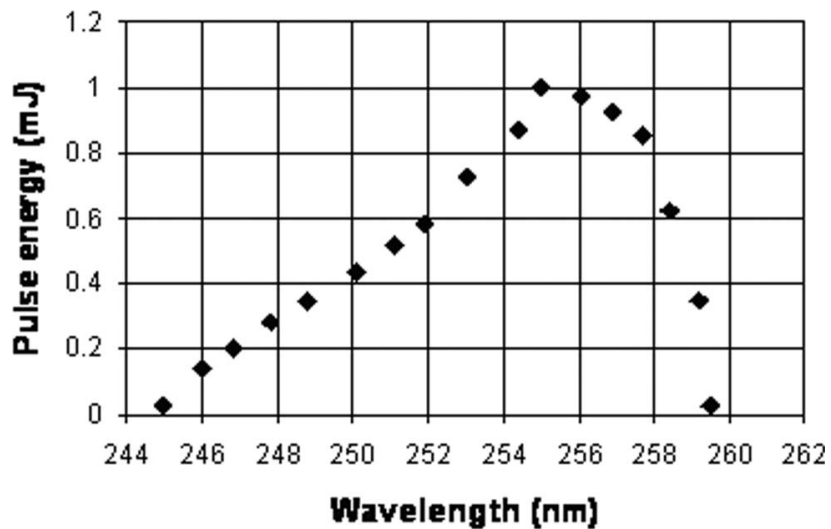

Fig. 5. Pulse energy of the UV-OPO as a result of a continuous wavelength tuning. 


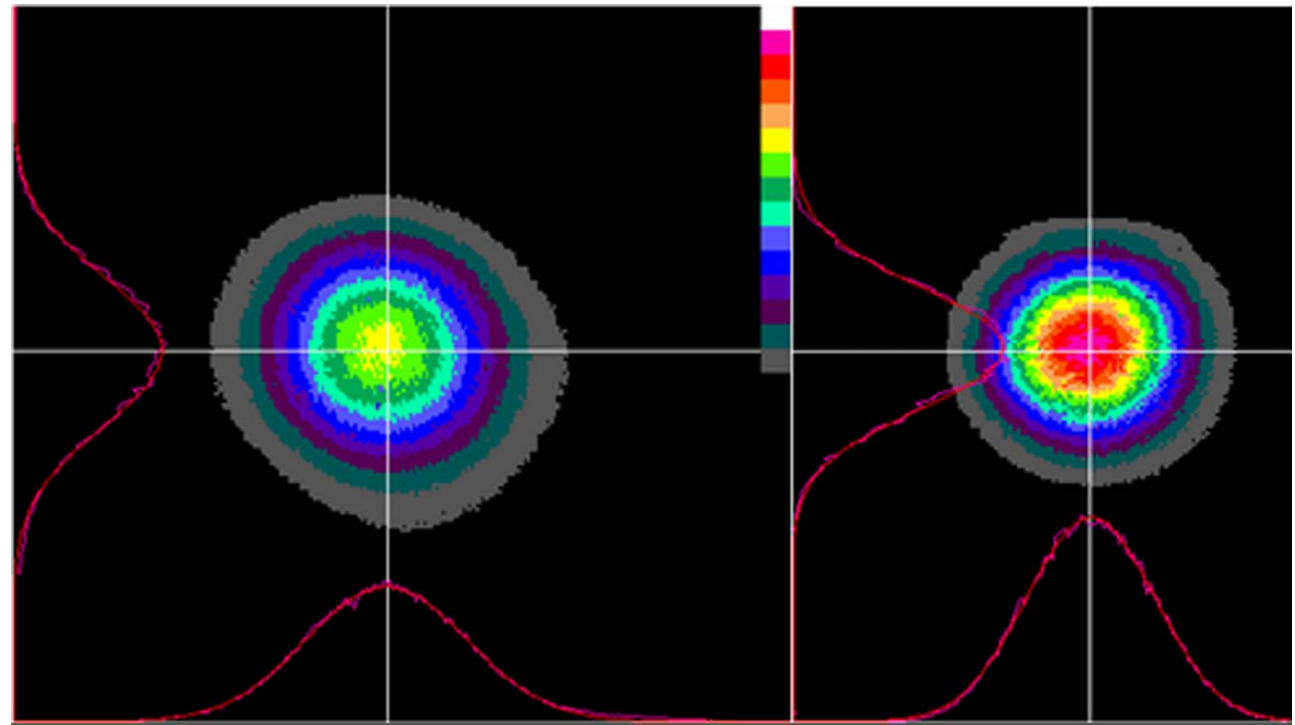

Fig. 6. (Color online) Results from measurements of the beam profiles of the UV OPO (left) and of the Nd:YAG MOPA (right).

are displayed together with the corresponding Gaussian fit curves, by which the quality of the UV beam is still underlined.

\section{Conclusions}

We have demonstrated a compact, all-solid-state laser system that is capable of producing tunable UV radiation in the wavelength range of $250 \mathrm{~nm}$ with a high beam quality and a high overall electrical-tooptical efficiency of $0.25 \%$. The system's features of fiber pumping and passive $Q$-switching, as well as a low sensitivity on changes of the diode temperature, provide optimum conditions for a versatile, robust device of high practical usefulness at difficult environmental conditions, with low demands on temperature stabilization and heat dissipation. The robustness of the MOPA configuration with respect to misalignment, vibration, and temperature variation has been proven in the context of the development of a similar setup for operation in space [24]. Furthermore, another design goal of a low-powerconsuming device has been met, as the system is well suited to be operated by a commercial battery power supply, without any need of an active cooling unit. The main factor that currently limits the scaling of the pulse energy of the MOPA is the onset of parasitic lasing, when the pump intensity is further increased. This can be intercepted by distributing the pump power to more than one amplifier stage [22,24]. The same holds when the pulse repetition rate would be further increased, since thermal lensing and thermally induced stress strongly affect the beam quality, which must be optimal in order to achieve high efficiency for the subsequent nonlinear-optical processes.

Since the OPO was operated only up to a pump power of 2.3 times the threshold, the maximum pulse energy of the UV radiation, as well as the optical efficiency and the tuning range, would be significantly enhanced by increasing the power of the pump laser, e.g., to a value of $50 \mathrm{~mJ}$. Meanwhile, corresponding developments have been performed on a similar, compact MOPA system with two fiber-pumped amplifier stages [24]. The tuning range of the OPO could be further extended when resonator mirrors become available with optical coatings that would admit a broader wavelength interval for the OPO signal.

This work was sponsored by the Federal Ministry of Education and Research (BMBF), contract 13 N8100 (Project MILAN). The authors thank M. Schmidt and M. Sellhorst of Verein Deutscher Ingenieure (VDI), Düsseldorf, for their dedicated, administrative support, as well as G. Reithmeier and P. Zeller (EADS) for technical support.

\section{References}

1. A. Fix, M. Wirth, A. Meister, G. Ehret, M. Pesch, and D. Weidauer, "Tunable ultraviolet optical parametric oscillator for differential absorption lidar measurements of tropospheric ozone," Appl. Phys. B 75, 153-163 (2002).

2. D. M. Lubman, ed., Lasers and Mass Spectrometry (Oxford U. Press, New York, 1990).

3. P. Misra and M. A. Dubinskii, eds., Ultraviolet Spectroscopy and UV Lasers, Vol. 30 of Practical Spectroscopy Series (CRC Press, 2002).

4. C. Illenseer, H.-G. Löhmannsröben, and R. Schultze, "Application of laser-based ion mobility (IM) spectrometry for the analysis of polycyclic aromatic compounds (PAC) and petroleum products in soils," J. Environ. Monitor. 5, 780-785 (2003).

5. S. Imai, T. Yamada, Y. Fujimori, and K. Ishikawa, "Thirdharmonic generation of an alexandrite laser in $\beta-\mathrm{BaB}_{2} \mathrm{O}_{4}$," Appl. Phys. Lett. 54, 1206-1208 (1989).

6. J. Lublinski, M. Müller, F. Laeri, and K. Vogler, "Collinear and non-collinear sum-frequency mixing in $\beta-\mathrm{BBO}$ for a tunable 195-198 nm all-solid-state laser system,” Appl. Phys. B 61, 529-532 (1995)

7. S. B. Mirov, V. V. Fedorov, B. Boczar, R. Frost, and B. Pryor, "All-solid-state laser system tunable in deep ultraviolet based on sum-frequency generation in CLBO," Opt. Commun. 198, 403-406 (2001). 
8. M. Funayama, K. Mukaihara, H. Morita, T. Okada, N. Tomonaga, J. Izumi, and M. Maeda, "Continuously tunable coherent source over $202-3180 \mathrm{~nm}$ based on a Ti:sapphire laser," Opt. Commun. 102, 457-460 (1993).

9. T. Meguro, T. Caughey, L. Wolf, and Y. Aoyagi, "Solid-state tunable deep-ultraviolet laser system from 198 to $300 \mathrm{~nm}$," Opt. Lett. 19, 102-104 (1994).

10. D. J. Binks, P. S. Golding, and T. A. King, "Compact all-solidstate high repetition rate tunable ultraviolet source for airborne atmospheric gas sensing," J. Mod. Opt. 47, 18991912 (2000).

11. J. Sakuma, K. Deki, A. Finch, Y. Ohsako, and T. Yokota, "All-solid-state, high-power, deep-UV laser system based on cascaded sum-frequency mixing in $\mathrm{CsLiB}_{6} \mathrm{O}_{10}$ crystals," Appl. Opt. 39, 5505-5511 (2000).

12. F. Huang, Q. Lou, T. Yu, J. Dong, B. Lei, and Y. Wie, "Tunable solid state UV laser," Opt. Laser Technol. 33, 111-115 (2001).

13. K. A. Elsayed, S. Chen, L. B. Petway, B. L. Meadows, W. D. Marsh, W. C. Edwards, J. C. Barnes, and R. J. DeYoung, "High-energy, efficient, $30 \mathrm{~Hz}$ ultraviolet laser sources for airborne ozone-lidar systems," Appl. Opt. 41, 2734-2739 (2002).

14. A. V. Kachynski, V. A. Orlovich, A. A. Bui, V. D. Kopachevsky, A. V. Kudryakov, and W. Kiefer, "All solid-state pulsed ultraviolet laser widely tunable down to $188.5 \mathrm{~nm}$," Opt. Commun. 218, 351-357 (2003).

15. J. F. Pinto, L. Esterowitz, and G. H. Rosenblatt, "Frequency tripling of a $Q$-switched Cr:LiSAF laser to the UV region," IEEE J. Sel. Top. Quantum Electron. 1, 58-61 (1995).

16. A. Borsutzky, R. Brünger, and R. Wallenstein, "Tunable UV radiation at short wavelengths $(188-240 \mathrm{~nm})$ generated by sum frequency mixing in lithium borate," Appl. Phys. B 52, 380 (1991).

17. B. Wu, F. Xie, C. Chen, D. Deng, and Z. Xu, "Generation of tunable coherent vacuum ultraviolet radiation in $\mathrm{LiB}_{3} \mathrm{O}_{5}$ crystal," Opt. Commun. 88, 451-454 (1992).

18. I. Horn, D. Günther, and M. Guillong, "Evaluation and design of a solid-state $193 \mathrm{~nm}$ OPO-Nd:YAG laser ablation system," Spectrochim. Acta Part B 58, 1837-1846 (2003).

19. D. J. Armstrong and A. V. Smith, "All solid-state highefficiency tunable UV source for airborne or satellite-based ozone DIAL systems," IEEE J. Sel. Top. Quantum Electron. 13, 721-731 (2007).

20. M. Tiihonen, V. Pasiskevicius, and F. Laurell, "Tailored UVlaser source for fluorescence spectroscopy of biomolecules," Opt. Lasers Eng. 45, 444-449 (2007).

21. A. Fix and G. Ehret, "Intracavity frequency mixing in pulsed optical parametric oscillators for the efficient generation of continuously tunable ultraviolet radiation," Appl. Phys. B 67, 331-338 (1998).

22. P. Peuser, W. Platz, P. Zeller, T. Brand, M. Haag, and B. Köhler, "High-power, longitudinally fiber-pumped, passively $Q$ switched Nd:YAG oscillator-amplifier," Opt. Lett. 31, 19911993 (2006).

23. S. Forget, F. Balembois, P. Georges, P.-J. Devilder, "A new 3D multipass amplifier based on Nd:YAG or Nd:YVO 4 crystals," Appl. Phys. B 75, 481-485 (2002).

24. D. Kracht, S. Hahn, R. Huss, J. Neumann, R. Wilhelm, M. Frede, and P. Peuser, "High efficiency, passively $Q$-switched Nd:YAG MOPA for spaceborne laser-altimetry," Proc. SPIE 6100, 610021 (2006). 